\title{
In situ observation of the evolution of porous silicon interference filter characteristics
}

\author{
J. Volk ${ }^{*}, 1$, K. Ferencz ${ }^{2}$, J. J. Ramsden ${ }^{3}$, A. L. Tóth ${ }^{1}$, and I. Bársony ${ }^{1}$ \\ ${ }^{1}$ Research Institute for Technical Physics and Materials Science MFA, P.O. Box 49, 1525 Budapest, \\ Hungary \\ ${ }^{2}$ Research Institute for Solid State Physics and Optics SZFKI, P.O. Box 49, 1525 Budapest, Hungary \\ ${ }^{3}$ Department of Advanced Materials, Cranfield University, MK43 0AL, UK
}

Received 10 June 2004, revised 13 September 2004, accepted 27 January 2005

Published online 8 June 2005

PACS 42.79.Ci, 78.30.Am, 82.45.Vp

Porous silicon multilayer formation was observed by in situ monitoring of the reflectivity spectra in the visible range. In order to reproduce the formation process optical model simulation was carried out. For demonstration of this method a 24-layer microcavity structure was selected. Although in this low wavelengths region some absorption and scattering effects complicate the overall picture, the combined analysis throws new light upon the evolution of the porous silicon multilayer.

(c) 2005 WILEY-VCH Verlag GmbH \& Co. KGaA, Weinheim

\section{Introduction}

Porous silicon is a very attractive material for the preparation of multilayered stacks (PSM), forming multilayer Bragg reflectors (BR) or Fabry-Perot (FP) multilayer cavities [1]. The attractive optical performance of such passive elements, realised by the sequence of high and low porosity layers stacked upon each other, originates from the use of the interference principle. Reece et al. proved that a high quality PS microcavity with subnanometre linewidth is achievable [2]. These structures have diverse applications, especially in the field of sensors, where accurately tailored optical performance is essential. The aim of this work was to monitor the evolution of the reflextion curve during PSM formation, obtaining information about the formation mechanisms and some relevant structural and processing quantities (e.g. porosity and etching rate). Contrary to the general practice of designing and preparing PS interference filters for the near infrared range, we considered visible wavelengths in order to facilitate the in situ, real time high resolution reflectometry studies. The simulation of such multilayer structures, however, has to take the imaginary part of the index of refraction also into consideration.

\section{Experimental}

PSM stacks were prepared at room temperature on highly doped 3-inch p-type Si wafers $(\rho=0.005 \Omega \mathrm{cm})$ by electrochemical etching in a 1:1 mixture of $48 \%$ aqueous $\mathrm{HF}$ and ethanol. Current densities of $8 \mathrm{~mA} / \mathrm{cm}^{2}$ and $350 \mathrm{~mA} / \mathrm{cm}^{2}$ were applied sequentially for $5.5 \mathrm{~s}$ and $0.8 \mathrm{~s}$, respectively to produce low and high porosity, i.e. high $(\mathrm{H})$ and low $(\mathrm{L})$ refractive index layers underneath each other. $15 \mathrm{~s}$ pauses were inserted before changing the current in order to homogenize the HF concentration in the electrolyte during formation [3].

"Corresponding author: e-mail: volk@mfa.kfki.hu, Phone: +36 1392 2694, Fax: +36 13922226 


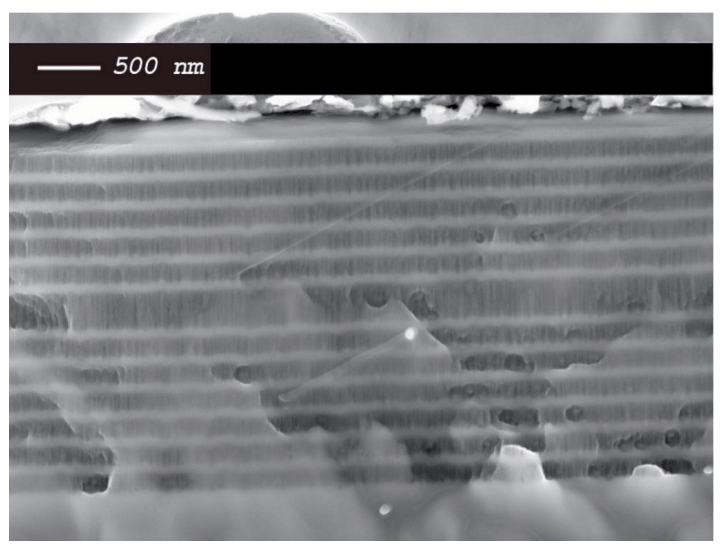

Fig. 1 SEM image taken on the prepared 24-layer FP structure. The cross section was cut by ion milling, the artifacts are the result of resputtering. Still, the perfect periodicity of the PSM structure is obvious.

The realized PSM stack was a 24-layer FP interference filter (in Fig. 1) having an optical structure of ambient-[HL] ${ }^{5} \mathrm{HLL}[\mathrm{HL}]^{6}$-substrate. Full reflexion spectra were taken by an Avaspec 2048 spectrometer once per second in the range of $\lambda=400-1000 \mathrm{~nm}$. The fast recording was provided by a Si based CCD detector arrangement. The acquisition of the reflected light was achieved with a reflexion probe with input and output optical fibres inside an HF protection PC test tube. It was immersed into the HF electrolyte and held at a constant distance of $10 \mathrm{~mm}$ from the sample surface. Figure 2 shows the experimental setup (a) and a typical evolution spectrum (b).

For the analysis we considered the whole process as $12 \mathrm{HL}$ periods, where the 6-th period is altered due to the resonator (HLL). From the point of view of the in situ reflectometry the measurement and the electrochemical etching were synchronized. Three spectra were recorded per HL period, i.e. they corresponded to the status of anodic etching at the bottom of the forming PSM: H/2, H, and HL. This means that e.g. the 28-th recording corresponds to the layer sequence starting from the wafer surface according to the optical model of $[\mathrm{LH}]^{5} \mathrm{HLL}[\mathrm{HL}]^{3} \mathrm{H} / 2$, i.e. to the PSM of $91 / 3$ periods. Optical model analysis was carried out by well-known thin film calculation techniques [4]. The complex refractive indices of the constituent layers were calculated with the Bruggeman effective medium approximation from the porosity data [5]. The calculations had to take the presence of electrolyte in the pores into consideration, i.e. the unknown refractive properties of the HF-containing fluid. In a first approximation in this work pure ethanol was considered as the filling medium in the model, and was also used as the ambient medium for the description of the submerged PSM.

(a)

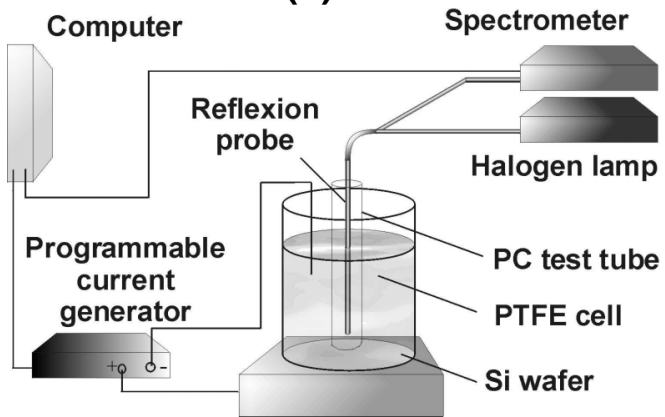

(b)

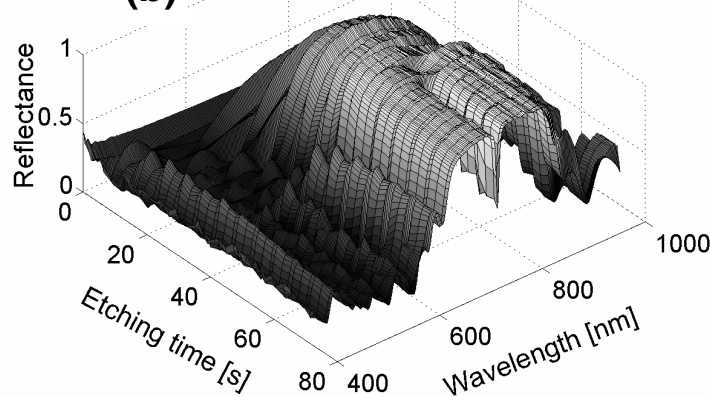

Fig. 2 Anodization setup with the inserted reflexion probe in the PC test tube for the in situ reflectometry (a), and the $3 \mathrm{D}$ representation of the measured spectra vs. etching time (b). 


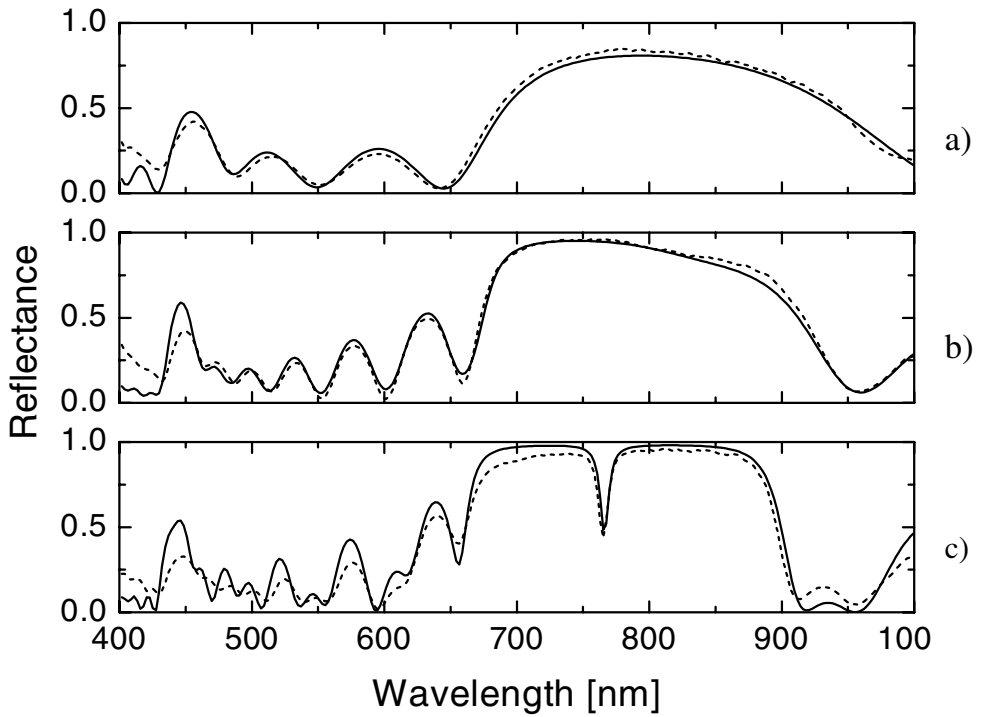

Fig. 3 Three characteristic simulated (dotted) and measured (solid) reflectance spectra taken after the completion of 3(a), 6(b) and 9(c) periods, respectively. The formation of the resonator was completed after the 5,5 period in spectrum (b), the associated absorption peak is only visible here in (c), and further deepens as etching progresses.

\section{Results and discussion}

Figure 3 shows the comparison of three reflectance spectra taken after the completion of 3, 6 and 9 periods (simulated and measured), respectively. The measured curves follow the predictions, the maximum reflectance increases with increasing number of HL periods. After the 6-th period (in Fig. $3 \mathrm{c}$ ) a resonant dip develops in the middle of the high reflectance region due to the microcavity. The FWHM value of this absorption peak decreases from ca $20 \mathrm{~nm}$ to $6 \mathrm{~nm}$ during the formation of the Bragg mirror beneath the microcavity.

In order to extract the optical parameters from the in situ monitoring data the arbitrarily selected snapshots were fitted by an optical model in Fig. 3. The porosity and thickness data were calculated from the optical model for all three situations in Fig. 3. In the model a pair of average porosity values was considered for each case, as the layers were coupled in the PSM. There was, however, a clear indication of average porosity increase in the already etched PSM during the subsequent processing. This can be interpreted in terms of chemical and photochemical etching. The latter is particularly pronounced in this case, as the in situ reflectometry involves intense illumination of the analysed spot. The porosity ranged between 39 to $43 \%$ for the high refractive index layer and between 68 and $76 \%$ for the low refractive index layer.

The in situ experimental and calculated data for the whole preparation process is compared in Fig. 4. The experimental 2D reflectivity map was plotted by collecting the 36 reflectivity snapshots in the evolving PSM stack. The position of the reflected intensity maximum in the top Bragg mirror (up to $6 \mathrm{HL}$ pairs) and the resonant peak position (at the higher number of HL pairs) is blue shifted. As expected, the optical performance of the mirror deteriorates transiently (see grey scale) until the HL period actually being etched, had been completed. The evolution of the stop bands has a break point at the HL pair number where the effect of the microcavity becomes dominant. At this position the stop bands get instantaneously broader. It is also obvious that the stop bands are getting more and more asymmetric with the evolution of the PSM. The reason for that is probably the increasing interface roughening in the bottom Bragg mirror, causing a larger scattering loss of the lower wavelengths. 

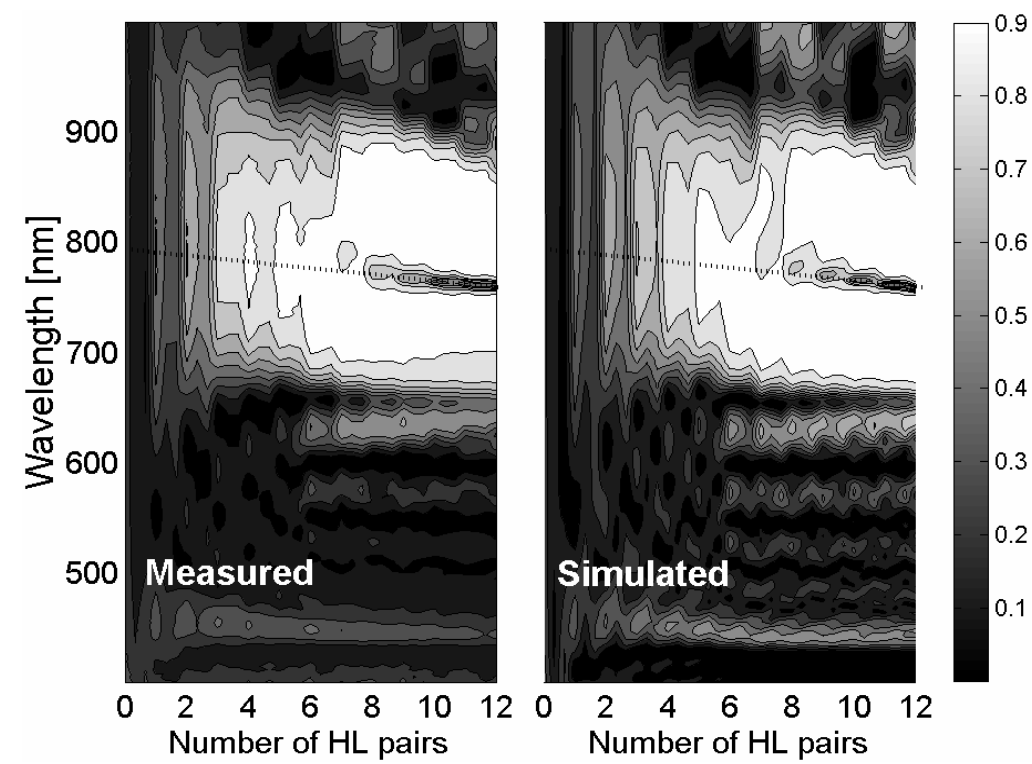

Fig. 4 2D reflectance map showing the evolution of the reflectivity spectra during multilayer development according to the measured (left) and calculated (right) performance. The magnitude of the obtained reflectivity is represented by the grey scale, plotted against the wavelength and the number of HL pairs etched within the stack.

In the simulation map from an optical model in Fig. 4 we assumed uniform propagation of the etching front. In all the individual steps the calculation was performed with constant average porosity values, which, however, increased with time. The side lobes, fringes at low wavelengths outside the stop band, are more significant in the simulation. In the measurement they are less visible, most probably due to the above mentioned scattering effect at the roughening interfaces at the bottom of the stack.

\section{Conclusion}

We have shown that in situ reflectivity monitoring of PSM formation is feasible. The measured data show excellent agreement with the optical model simulation. The obtained results suggest the possibility of direct feedback control of the anodization, which is essential for accurate tailoring of the required optical performance. This in situ investigation revealed the effect of illumination by the light source of the reflectometer, which caused slightly enhanced photochemical dissolution at the measured spot, which in turn caused a blue shift in the position of the resonance peak. The very same effect, however, can also be used for fine tuning resonance towards lower wavelengths in a given PSM design.

Acknowledgments This work has been supported by the grants of the Hungarian Scientific Research Foundation (OTKA) Nos. T033094 and T046696 and by the Royal Society Joint Project Grant entitled: "Fabry-Perot multilayers for chemical sensing". The authors acknowledge the assistance of Ms. M. Payer with sample preparation and Dr. Á. Barna with ion milling.

\section{References}

[1] S. Frohnhoff and M. G. Berger, Adv. Mater. 6, 963 (1994).

[2] P. J. Reece, G. Lerondel, W. H. Zheng, and M. Gal, Appl. Phys. Lett. 26, 4895 (2002).

[3] S. Billat, M. Thonissen, R. Arens-Fisher, M. G. Berger, M. Kruger, and H. Luth, Thin Solid Films 297, 22 (1997).

[4] H. A. Macleod, Thin-Film Optical Filters, 3rd edition, Ch. 2 (IoP, Bristol and Philadelphia, 2001).

[5] D. A. G. Bruggeman, Ann. Phys. 24, 636 (1935). 